\title{
An algorithm to optimize deployment of charging base stations for WRSN
}

\author{
Peng Wan, Yuhua Cheng ${ }^{*}$ (D, Baoyu Wu and Gaofeng Wang
}

\begin{abstract}
Optimizing deployment of charging base stations in wireless rechargeable sensor networks can considerably reduce the cost. Previously, the charging base stations are simply installed at some fixed special points (e.g., the grid points) after partitioning the area distributed by sensor nodes. In this paper, a new algorithm of planning the charging base stations is proposed based on the greedy algorithm and the location relationship of the sensor nodes. The proposed algorithm exploits the local search ability and avoids falling into an exponential increase of the number of the charging base stations (i.e., combinatorial explosion). The simulated results show that the proposed algorithm can result in less number and flexible deployed locations of the charging base stations. In addition, this algorithm provides a novel solution for the point coverage problems.
\end{abstract}

Keywords: Wireless rechargeable sensor network, Greedy algorithm, Charging base station

\section{Introduction}

The massive Internet of things (IoTs) [1] which provide big data for cloud computing [2-6] and traditional networks [7] have been applied to many fields in reality. As an important part of the IoT [8, 9], the wireless sensor network (WSN) [10-12] has the capabilities of data collection [13-15], data storage, and wireless communication $[16,17]$. WSN plays a significant role in the construction of smart cities [18, 19], smart agriculture [20], and public utility monitoring [21], etc. However, WSNs are traditionally powered by batteries [22], which result in a limit lifetime of the networks. Many researchers effectively extend the network life cycle by reducing energy consumption or improving the energy performance of sensor network [23-29]. A potential solution of power supply for the sensor nodes in WSNs is using wireless power transfer (WPT) technologies [30] to realize a wireless rechargeable sensor network (WRSN) where some charging base stations are dedicatedly deployed to provide the power permanently, or mobile charging devices are dispatched to approach and charge the sensor nodes.

\footnotetext{
* Correspondence: chengyh@hdu.edu.cn

College of Electronics and Information, Hangzhou Dianzi University, Hangzhou 310018, China
}

Although higher power transfer efficiency can be obtained for the near-field WPT than the far-field WPT, larger-sized coils and good alignment are required in reality for the near-field WPT because of the non-radiated magnetic coupling and radiated covering properties for the near-field and far-field WPT technologies, respectively. In practice, the far-field WPT with the property of radiation is more suitable for WRSN, because of the relatively large distance from the base station to the sensors, which is usually much larger than the size of the sensor nodes (especially under the requirement of compact size for sensor nodes).

Existing approaches on WRSN charging can be divided into two types, i.e., the mobile charging strategy and the static charging strategy. The main optimization goal of the mobile charging strategies [31-36] is to reduce the charging process cost (e.g., the number of the mobile charging devices such as the charging vehicles and the charging time) as much as possible while ensuring that the sensors can work continuously. Although the mobile charging strategy looks like more flexibility, it is not automatic enough and depends on the dispatch algorithm. In addition, the mobile charging strategy usually relies on the near-field WPT and short charging time is assumed. However, the environment (e.g., the accessible distance) of the distributed sensors will prevent the 
charging devices from charging the sensors effectively because of the restriction of the sizes and directions of the transmitting and receiving coils for the near-field WPT. Because of the advantage of convenience and stability, the static charge strategy is chosen in many applications, at which this paper is focused.

For the static charge strategy, the authors of [37] optimized the number of the charging base stations by considering the point provisioning and path provisioning problems. However, the work of [37] is essentially similar to the traditional wireless network coverage problem [38], and only the case of the uniformly distributed sensor nodes is discussed. The authors of [39] proposed a redundancy algorithm, which is essentially an improved simulated annealing algorithm, to optimize the number of charging base stations. However, the simulation validation does not consider the power consumptions of the sensor nodes in [39], which are actually related to the charging distance and charging efficiency in reality. The authors of [40] proposed an improved firefly algorithm to solve the multi-objective optimization problem. However, only an example with few sensor nodes is taken in [40]. The authors of [41] placed the wireless charging base stations on a network grid point after uniformly portioning the area where the sensors were distributed. Under the assumption of the base station with a certain height and a fixed radiation angle, a node-based greedy cone selecting algorithm and a pair-based greedy cone selecting algorithm were proposed. Furthermore, the adaptive pair of the greedy cone selection (APB-GCS) algorithm was proposed in [42] based on the algorithms in [41], which is suitable for the case with more sensors. However, the numbers of the base stations in [41, 42] are insufficiently optimized, although the planning was simpler, since the sensors' distributed area was partitioned uniformly and the charging base stations were fixed at the grid points. In addition, the number of the charging base stations and the complexity of the algorithm depend on the size of the cells after portioning. In reality, the charging base stations can be optimized at better positions, not only at some special points (e.g., the grid points). The deployment problem of charging base station is obviously a non-convex optimization problem [43] which has been studied for wireless sensor networks [44-48]. The local optimal solution of this problem is not necessarily the global optimal solution actually. In this paper, a new algorithm, called the charging circle-greedy (CCG) algorithm by combining both the merits of the nodes' location consideration and the charging area partition, is proposed to achieve better optimization. Multiple simulations are also given for validations in this paper.

\section{CCG algorithm method}

\subsection{WRSN network model}

The number of the wirelessly rechargeable sensor nodes are assumed to be $N$ and denoted as $U=\left\{u_{1}, u_{2}, u_{3} \ldots u_{N}\right\}$, which are randomly distributed in the two-dimensional region, $\Omega$. The omnidirectional charging base stations, each of which can charge a circular area with $R$-radius, denoted as $S=\left\{s_{1}, s_{2}, s_{3} \ldots s_{M}\right\}$, where $M$ is the number of charging base stations. The charging base station $s_{i}$ is actually a subset of $U$, as shown in Fig. 1 where the charging base station $s_{i}$ is also a set including more than one node. Due to the power transfer efficiency decreases with the power transfer distance, the effective coverage area is limited to a circle with $R$-radius for each omnidirectional radiated charging base station. The sensor nodes out of this $R$-radius circle cannot receive effective power. One sensor node may be covered by multiple charging base stations and receives power from multiple charging base stations simultaneously. Some sensor nodes need to be covered by multiple charging base stations to satisfy their power consumptions.

\subsection{Problem definition}

The problem is defined as a minimum number of charging base stations that are required to provide enough power to the $N$ randomly distributed charging sensor nodes in the two-dimensional region $\Omega$. The coverage range of a charging base station is a circle with radius $R$. One sensor node can be covered by multiple charging base stations according to its power consumption. Building some base stations to cover many discrete sensor nodes in a plane region [41] is a nonlinear programming problem, which are basically NP-hard problems $[49,50]$. The NP-hard problems can be solved only by approximation methods.

\subsection{Methods}

If each sensor node is required to be covered only once by any charging base station, the deployment problem of the charging base station is translated to a point coverage problem [51]. If the locations of the sensor nodes are unknown, the total area should be covered by the charging base stations. Assuming that $N$ sensor nodes are randomly distributed in the $H \times L$ square region $\Omega$ as shown in Fig. 1, $M_{\max }$ charging base stations are required to cover all the sensors once. That is, there is no dead zone coverage in the region $\Omega$.

$$
M_{\max }=\left\lceil\frac{H}{\sqrt{2} R}\right\rceil \times\left\lceil\frac{L}{\sqrt{2} R}\right\rceil
$$

where the largest inscribed square of a circle covered by a base station is $2^{0.5} R$. If each sensor node is only required to be covered once, $M_{\max }$ in (1) is actually the 


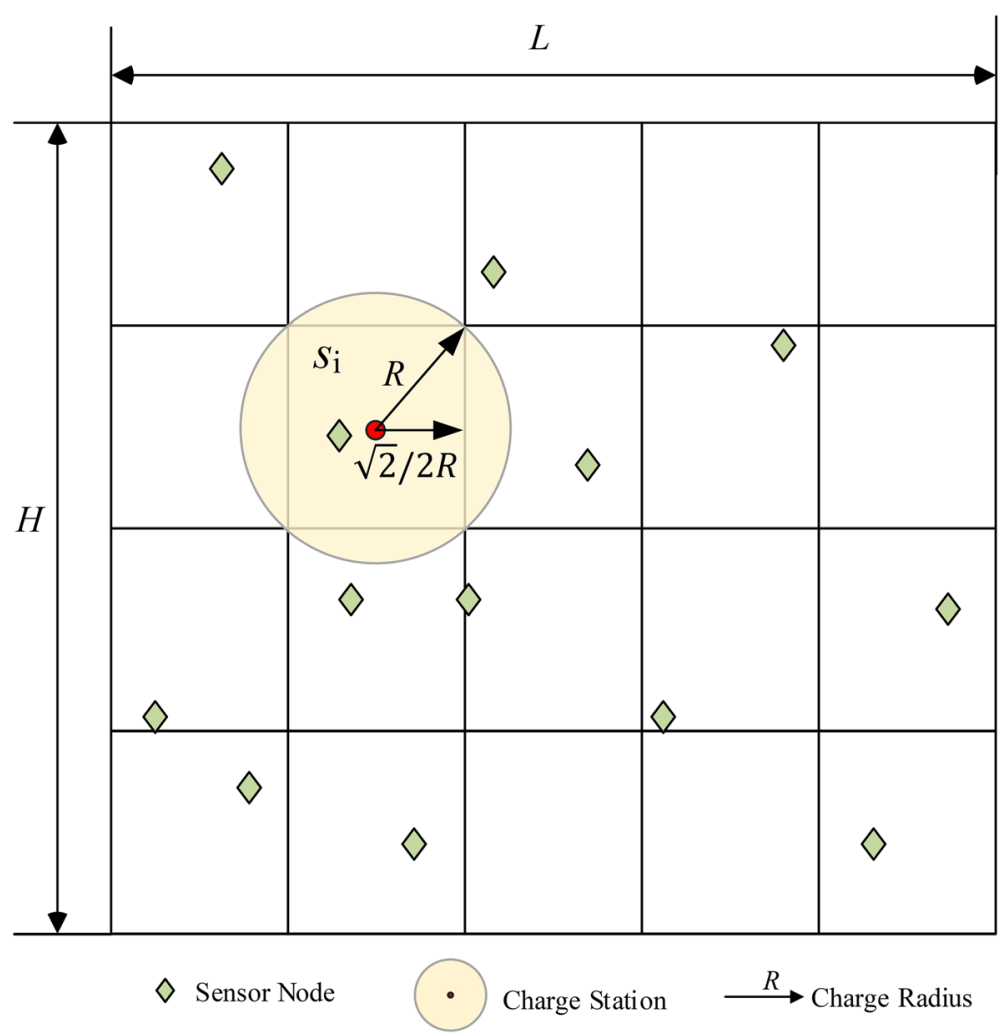

Fig. 1 Model of WRSN networks

upper limit of $M$, regardless of the number of sensor nodes in the area $\Omega$.

In practice, the location information of the sensor nodes can be obtained. The sensor nodes can be combined according to their topology information. Each combination contains the sensor nodes which can be covered by a charging base station. In this situation, the increase of the sensor nodes (especially when the sensor nodes are crowded) leads to the exponential increase of the charging base stations, because a charging base station exists potentially as long as the distance between arbitrary two sensor nodes is shorter than $2 R$. Selecting the optimal set of charging base stations from the order of exponential numbers by using the existing methods [39] or the ergodic method to find the optimal solution will result in combinatorial explosion problem [52], consequently, exceeding $M_{\max }$ for the number of base stations and non-polynomial time complexity $[53,54]$. In actual, this is similar to the optimization version of a set cover problem [55] which is a NP-hard problem.

In this paper, a new algorithm based on greedy algorithm is proposed. The greedy algorithm has the advantage of finding a local optimal solution and has the disadvantage of deficiencies in the overall search ability which is optimized here by setting a travel direction for the greedy circle in the greedy algorithm. Consequently, exponentially increasing the number of candidate base stations as the number of the sensor node increases is prevented and the number of charging base stations is optimized. As shown in Fig. 2, the region $\Omega$, a two-dimensional coordinate system, is divided into $T$ rectangles with $2 R$-height and $L$-width, where $T=\lceil H /$ $(2 R) 7$. The set of the sensor nodes, whose locations are known, distributed in the region $y \in[2(i-1) R, 2 i R]$ interval is denoted as $Q_{i}$, and the total set of the sensor nodes is $Q=\left\{Q_{1}, Q_{2} \ldots Q_{T}\right\}$.

The specific steps of the CCG algorithm are as follows:

Step 1: Classify all the sensor nodes $u_{i}(i=1,2 \ldots N)$ according to their $y$-coordinate values and store them into $Q_{i}$ in which all the $y$-coordinate values belongs to $[2(i-1) R, 2 i R]$. The total set of the sensor nodes is $Q=\left\{Q_{1}, Q_{2} \ldots Q_{T}\right\}$

Step 2: Let $i=1$;

Step 3: Find the sensor nodes with the smallest $x$ coordinate value in $Q_{i}$, denoted as $u_{k}$;

Step 4: Find a set of sensor nodes whose distances from $u_{k}$ are not greater than $2 R$, denoted as $s_{k}$; Step 5: List the sensor node pair whose distance is greater than $2 R$ in $s_{k}$, and exclude one of the sensor 


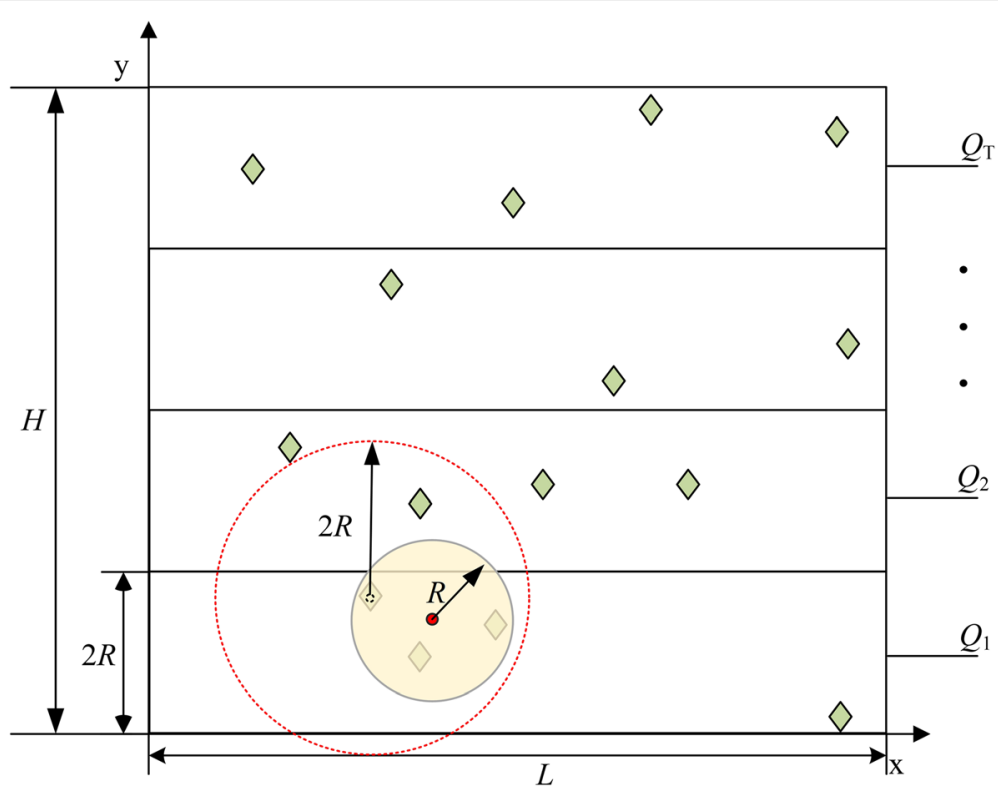

Fig. 2 Diagram of the procedure of CCG algorithm

nodes in this node pair from $s_{k}$; the excluded priority: the node not located in $Q_{i}>$ the node with a higher $x$ coordinate value in $Q_{i}>$ the node $u_{k}$;

Step 6 Exclude some of the nodes in $s_{k}$ until all nodes in $s_{k}$ can be covered by a circle with radius $R$; the excluded priority: the sensor node not located in $Q_{i}>$ the node with a higher $x$-coordinate value in $Q_{i}>$ the node $u_{k}$;

Step 7: Store $s_{k}$ in $S$; in $s_{k}$, exclude the sensor nodes satisfying the number of coverage (i.e., satisfying the power replenishment) from $Q$ and leave the sensor nodes not satisfying the number of coverage in $Q$; and repeat step 3 through step 7 until $Q_{i}=\emptyset$; and Step 8: Let $i=i+1$, repeat step 3 to step 8 until $Q=\emptyset$, and end the algorithm.

In these specific steps, step 4 is to find the core point $u_{k}$. Taking $u_{k}$ as the center of a circle with $2 R$-radius, the sensor nodes covered by this circle are stored in the set $s_{k}$. As an example, the result after step 4 is the circle with dotted line in Fig. 2. Step 5 cannot guarantee that all the sensor nodes in the $s_{k}$ are covered by a charging base station with a radius $R$, although the distances between any two sensor nodes is smaller than $2 R$. That is, another step, i.e., step 6 , is required. As an example, the result after step 6 is the circle with a solid line in Fig. 2. Step 6 is essentially finding out the minimum bounding circle of all sensors in the set $s_{k}$. If the radius of the smallest bounding circle is larger than $R$, some sensors in the set $s_{k}$ need to be excluded. The following describes the specific operations of step 6 : a) Find the two sensors which are the farthest away each other in the set $s_{k}$, denoted as $p_{1}$ and $p_{2}$; find the midpoint $c_{i}$ of $p_{1}$ and $p_{2}$; find the sensor node in the set $s_{k}$ that is the farthest from $c_{i}$ (except $p_{1}$ and $p_{2}$ ) in the set $s_{k}$ and denoted as $p_{3}$; judge whether the distance between $p_{3}$ and $c_{i}$ is greater than $R$ : if it is, enter step b; otherwise, go to step d;

b) Take sensor $p_{1}, p_{2}$, and $p_{3}$ as the vertices and make a triangle where $c_{i}$ updates the outer center of this triangle; if the distance between sensors and $c_{i}$ in $s_{k}$ is greater than $R$, enter step c; otherwise, go to step $\mathrm{d}$;

c) If only one sensor of $p_{1}, p_{2}$, and $p_{3}$ does not belong to $Q_{i}$, remove the sensor that does not belong to $Q_{i}$ from the set $s_{k}$; if two sensors in $p_{1}$, $p_{2}$, and $p_{3}$ do not belong to $Q_{i}$, then remove the sensor with larger $x$-coordinate value in this two sensors from the set $s_{k}$; otherwise, remove the sensor with the largest $x$-coordinate value in this three sensors $\left(p_{1}, p_{2}, p_{3}\right)$ from the set $s_{k}$; return step a; and

d) Put the set $s_{k}$ into set $S$, where $c_{i}$ is the location of the base station.

\section{Results of simulation}

Our proposed algorithm is firstly compared with the pair-based greedy cone selection (PB-GCS) algorithm, the node-based greedy cone selection (NB-GCS) algorithm in [41], and the adaptive pair-based greedy cone selection (APB-GCS) in [42], all of which are greedy algorithms. In [41, 42], the charging base station is fixed at a grid point which is $2.3 \mathrm{~m}$ above the ground. The coverage range of the charging base station is a cone with a busbar of $3 \mathrm{~m}$ as shown in Fig. 3. The sensor 


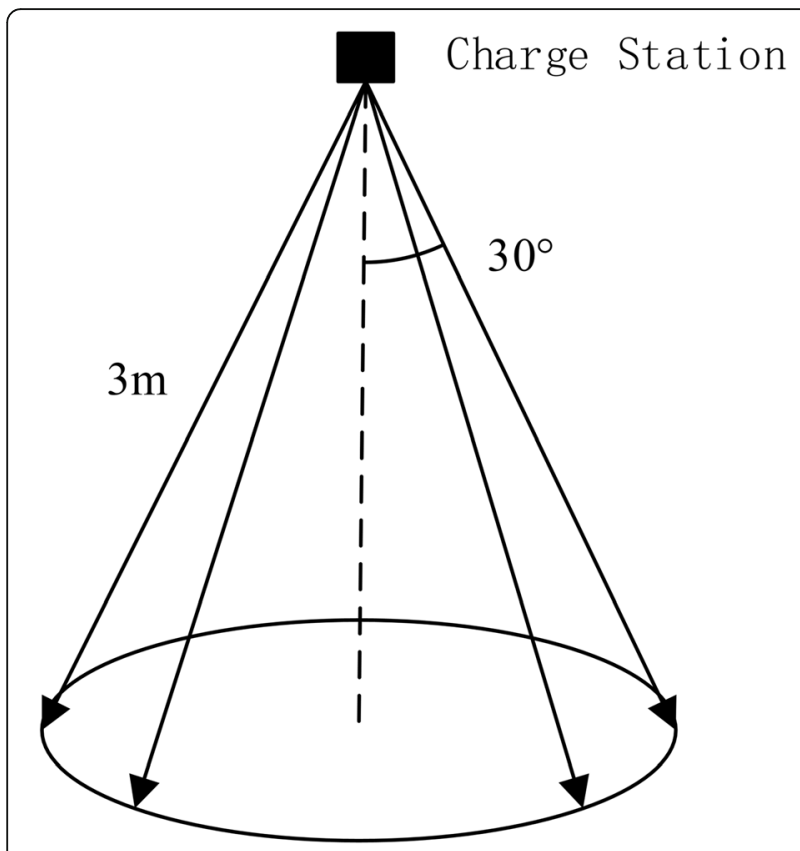

Fig. 3 Model of charging base station used in [41, 42]

nodes are randomly distributed (as an example) on a plane of $20 \mathrm{~m} \times 15 \mathrm{~m}$. Because the cone (i.e., the charging base station) can be rotated horizontally or vertically around the vertex, it can be calculated that the charging base station has a circle with a radius of $1.33 \mathrm{~m}$ when the coverage area of the plane is the smallest. In the simulation of the CCG algorithm, a harsher condition is used, i.e., a circle with a radius of $1.32 \mathrm{~m}$ is used.

As an example, 100 sensor nodes are randomly distributed and all of them need to be covered only once to meet the power requirements. The simulated number of base stations obtained by the CCG algorithm is 31, as shown in Fig. 4. As the second example, some sensor nodes need to be covered multiple times to meet their power requirements. For simplicity, it is assumed that $80 \%$ of the 100 sensor nodes need to be covered only once, and the remaining $20 \%$ of the sensor nodes need to be covered twice. The simulated number of charging base stations obtained by the CCG algorithm is 38, as shown in Fig. 5 where the prismatic nodes are the sensor nodes that need to be covered twice.

The comparison results of the number of charging base stations between CCG, PB-GCS, NB-GCS, and APB-GCS algorithms are shown in Figs. 6 and 7, respectively, for the case of $100 \%$ nodes covered once and the case of $80 \%$ nodes covered once. In [41, 42], because the charging base stations are fixed at the grid points, different grid side length selections will result in different results. Here, in Figs. 6 and 7, the optimized results published in $[41,42]$ are used. The grid side length is noted in the legend in Fig. 6, e.g., "APB-GCS (1.8m)" means that the grid side length is $1.8 \mathrm{~m}$. The grid side length in Fig. 7 is 1 m. From Figs. 6 and 7, a lesser number of the charging base stations can be observed in all the cases.

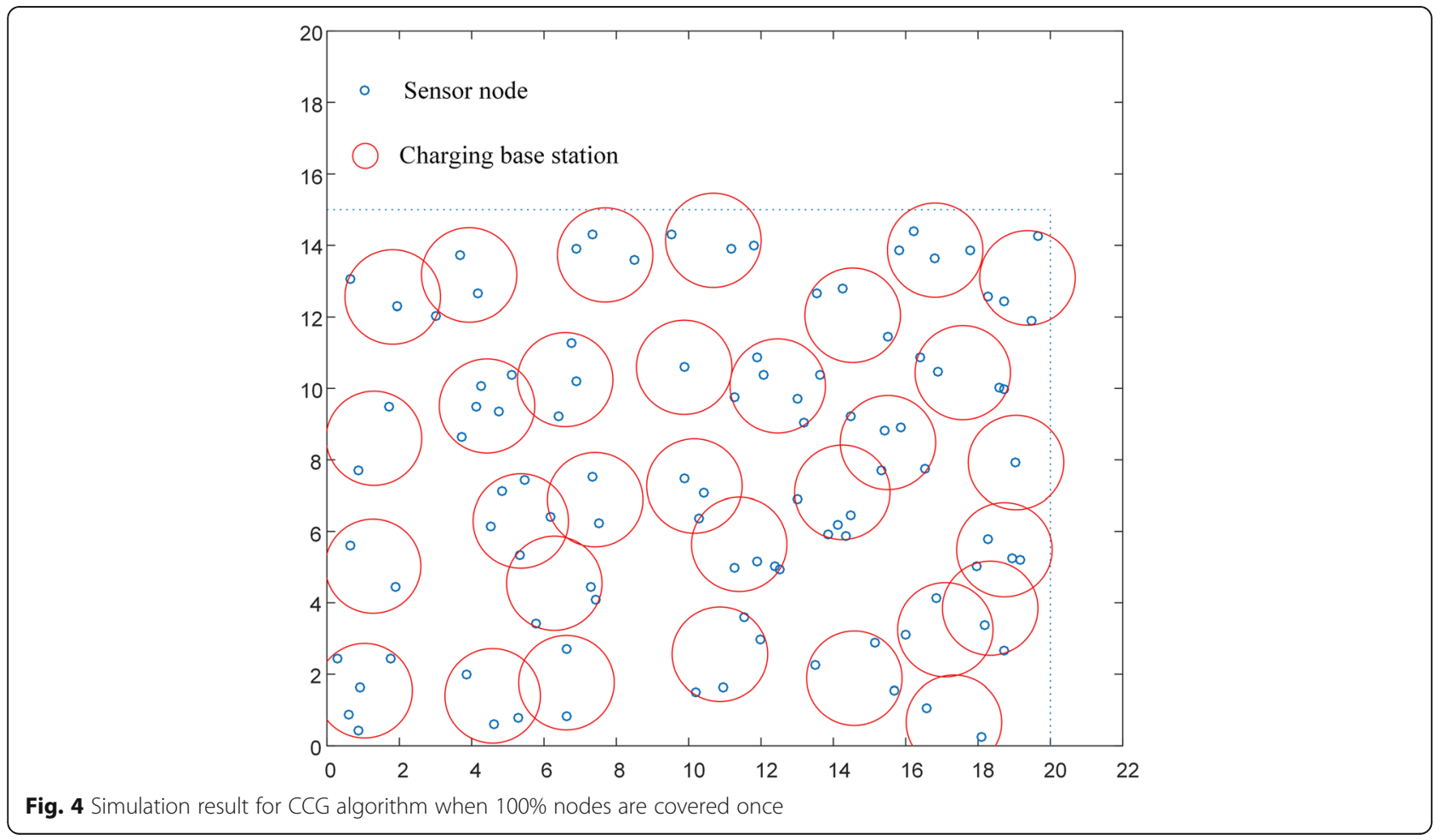




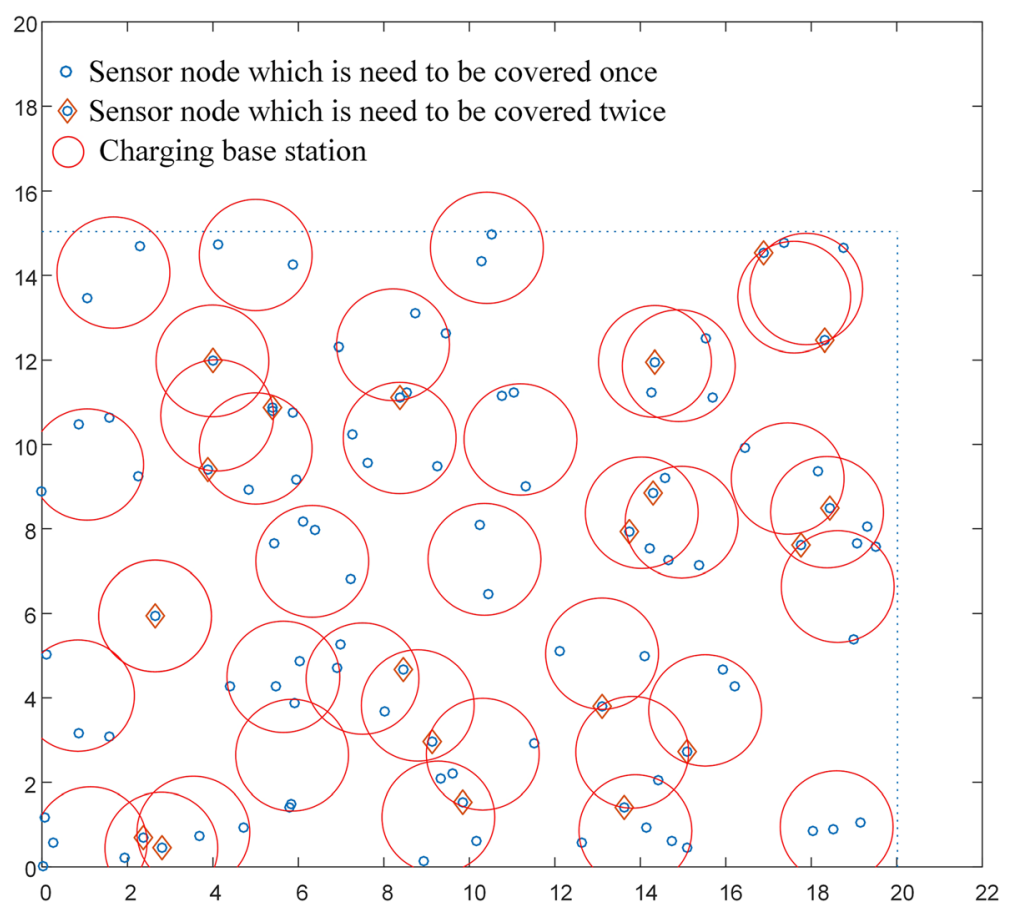

Fig. 5 Simulation result for CCG algorithm when 100\% nodes are covered once

The candidate number of the charging base station in the procedure of algorithms which reflects the computing complexity of the algorithms is compared as shown in Fig. 8. The results of the CCG algorithm are obviously lesser than the others. Additionally, in CCG algorithm, the positon of the charging base stations is not necessarily fixed to some special points

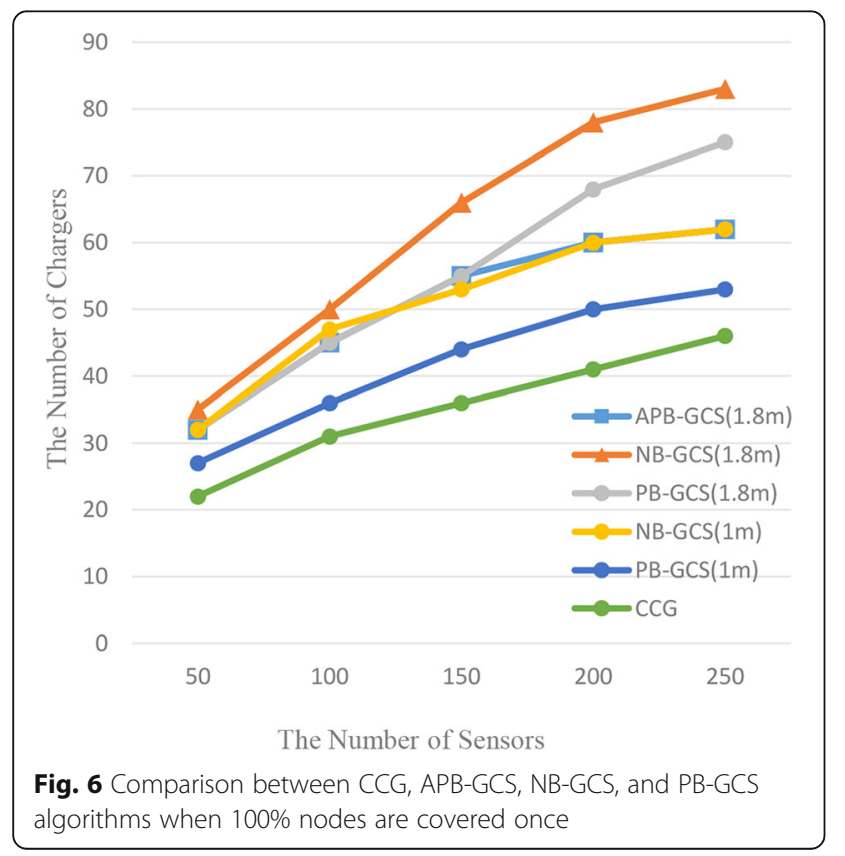

like other algorithms. The flexible positon advantage is important in reality.

Besides the comparison with the existing greedy algorithms, CCG algorithm is compared with two global algorithms such as simulated anneal-based charging (SABC) algorithm and layoff SABC (LSABC) algorithm [39] which are metaheuristic algorithms. Figure 9 shows the

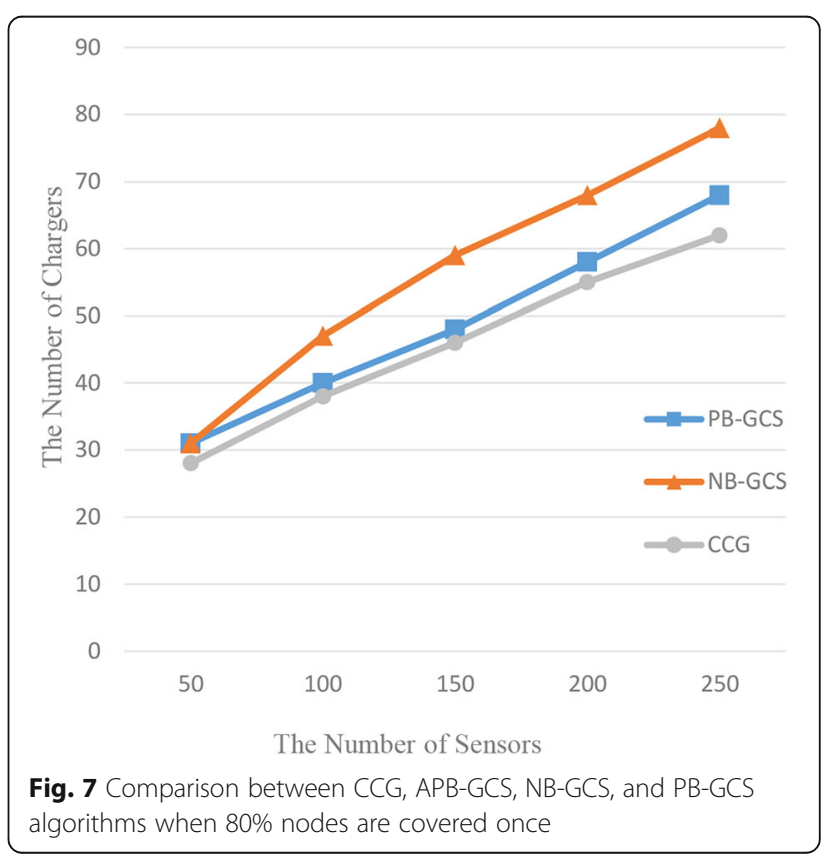




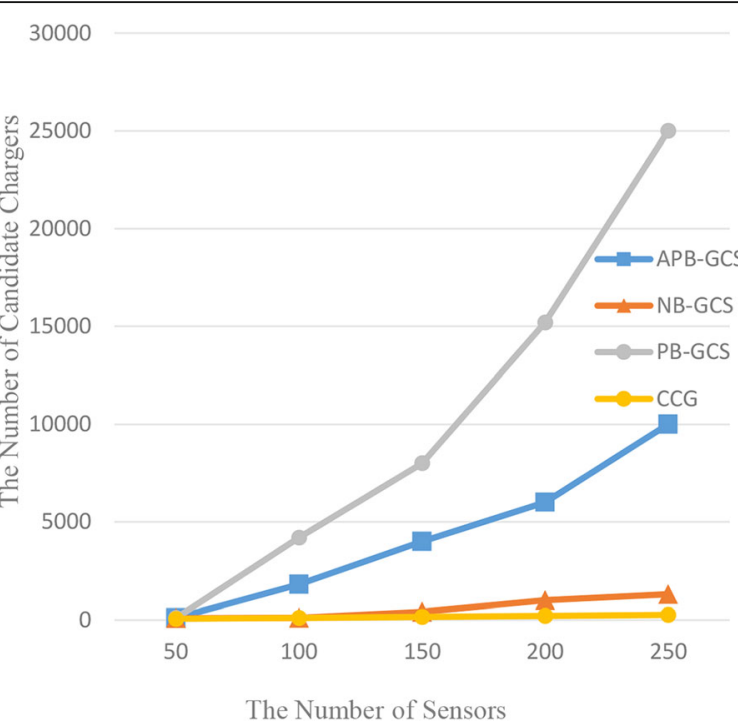

Fig. 8 Comparison of candidate charging base stations between CCG, APB-GCS, NB-GCS, and PB-GCS algorithms

comparison results of the number of charging base stations where the same parameters are set for the CCG, $\mathrm{SABC}$, and LSABC algorithms. The merit of less charging base stations for CCG algorithm can be observed in Fig. 9.

\section{Discussion}

From Figs. 6, 7, 8, and 9, the advantage in the number of charging base station for CCG algorithm has been shown. The complexity of the proposed algorithm is analyzed and discussed as follows:



Fig. 9 Comparison between CCG, SABC, and LSABC algorithms
(1) The algorithm can be considered as covering a bounded region $\Omega$ with multiple circles with an area of $\pi R^{2}$. When all the sensor nodes only need to be covered once, the maximum number of charging base stations is $M_{\max }$. That is, the CCG algorithm is convergent.

(2) A sorting algorithm is used in step 1 to partition the sensor nodes referring to the $y$-coordinate values. The time complexity of step 1 is $O(n)$. In step 3 , the worst case is that all the sensor nodes are in $Q_{i}$ and the corresponding complexity of step 3 is $O\left(n^{2}\right)$. In step 4 , if all the sensor nodes are clustered in $s_{i}$, the time complexity is $O\left(n^{4}\right)$, and the time complexity of the remaining steps are constants. So the time complexity of the CCG algorithm is $O\left(n^{4}\right)$. Actually, this is the worst time complexity in theory. In reality, all the nodes will not be distributed within the range of one base station. Therefore, the average time complexity of the CCG algorithm is commonly $O\left(n^{2}\right)$.

Aiming to the optimization target (i.e., the number of the charging base stations), the CCG algorithm has low complexity, is simple to use without lengthy formula derivation and complicated calculation, and has flexible deployment of the wireless charging base station. Actually, each sensor nodes consume different powers depending on the functions which are not considered comprehensively in this paper. In the future work, we will design a more efficient wireless charging base station deployment algorithm for the different power consumptions of the sensor nodes.

\section{Conclusions}

In order to optimize the deployment and the number of the charging base stations in wireless rechargeable sensor networks, the charging circle greedy algorithm has been proposed. This new algorithm makes use of the strong local search ability of the greedy algorithm and avoids the deficiency in the overall search ability by setting a travel direction for the greedy circle. Compared to the existing algorithms, the proposed algorithm can result in less number and flexible deployed locations of the charging base stations with lower time complexity. In addition, the charging circle greedy algorithm also inspires some new ideas for solving other point coverage problems.

\section{Abbreviations}

APB-GCS: Adaptive pair-based greedy cone selection; CCG: Charging circlegreedy; IoTs: Internet of things; LSABC: Layoff simulated anneal-based charging; NB-GCS: Node-based greedy cone selection; NP: Non-deterministic polynomial; PB-GCS: Pair-based greedy cone selection; SABC: Simulated anneal-based charging; WPT: Wireless power transfer; WRSN: Wireless rechargeable sensor network; WSN: Wireless sensor network

\section{Funding}

This work is supported by the following funding: Zhejiang Provincial Natural Science Foundation of China under grant No. LY17F010018 and the Natural Science Foundation of China under grant No. 61771175. 


\section{Availability of data and materials}

Not applicable.

\section{Authors' contributions}

YC and GW conceived the idea. PW and BW complete the algorithm. PW and $Y C$ wrote this paper. GW contributed to the reviewing of the manuscript. All authors read and approved the final manuscript.

\section{Competing interests}

The authors declare that they have no competing interests.

\section{Publisher's Note}

Springer Nature remains neutral with regard to jurisdictional claims in published maps and institutional affiliations.

Received: 3 July 2018 Accepted: 5 March 2019

Published online: 14 March 2019

\section{References}

1. X. Liu, S. Zhao, A. Liu, N. Xiong, A.V. Vasilakos, Knowledge-aware proactive nodes selection approach for energy management in internet of things. Futur. Gener. Comput. Syst. 92, 1142-1156 (2017)

2. B. Lin, W. Guo, N. Xiong, G. Chen, A.V. Vasilakos, H. Zhang, A pretreatment workflow scheduling approach for big data applications in multi-cloud environments. IEEE Trans. Netw. Serv. Manag. 13(3), 581-594 (2016)

3. Z. Wang, T. Li, N. Xiong, Y. Pan, A novel dynamic network data replication scheme based on historical access record and proactive deletion. J. Supercomput. 62(1), 227-250 (2012)

4. J. Yin, W. Lo, S. Deng, Y. Li, W. Z, N. Xiong, Colbar: a collaborative locationbased regularization framework for QoS prediction. Inf. Sci. 265, 68-84 (2013)

5. N. Xiong, J.W. AV Vasilakos, Y.R. Yang, A. Rindos, Y. Zhou, W.Z. Song, in 2012 IEEE 26th International Parallel and Distributed Processing Symposium. A selftuning failure detection scheme for cloud computing service (Shanghai, 2012), pp. 668-679

6. Y. Zhou, Y. Zhang, H. Liu, N. Xiong, A.V. Vasilakos, A bare-metal and asymmetric partitioning approach to client virtualization. IEEE Trans. Serv. Comput. 7(1), 40-53 (2014)

7. N. Xiong, A.V. Vasilakosb, L.T. Yang, C. Wang, R. Kannane, C. Chang, Y. Pan, A novel self-tuning feedback controller for active queue management supporting TCP flows. Inf. Sci. 180(11), 2249-2263 2010

8. K. Huang, Q. Zhang, C. Zhou, N. Xiong, Y. Qin, An efficient intrusion detection approach for visual sensor networks based on traffic pattern learning. IEEE Trans. Syst. Man Cybern. Part A Syst. Hum. 47(10), 2704 2713 (2017)

9. $\quad$ N. Xiong, R. Wen Liu, M. Liang, W. D, Z. Liu, W. H, Effective alternating direction optimization methods for sparsity-constrained blind image deblurring. Sensors 17(12), 174 (2017)

10. H. Zheng, W. Guo, N. Xiong, A kernel-based compressive sensing approach for mobile data gathering in wireless sensor network systems. IEEE Trans. Syst. Man Cybern. Part A Syst. Hum. 8(99), 1-13 2017

11. W. Guo, N. Xiong, A.V. Vasilakos, G. Chen, C. Yu, Distributed k-connected fault-tolerant topology control algorithms with PSO in future autonomic sensor systems. Int J of Sens Netw 12(1), 53-62 (2012)

12. Y. Zhou, D. Zhang, N. Xiong, Post-cloud computing paradigms: a survey and comparison. Tsinghua Sci. Technol. 22(6), 714-732 (2017)

13. Q. Zhang, Q. Qiu, G. We, K. Guo, N. Xiong, A social community detection algorithm based on parallel grey label propagation. Comput. Netw. 107(1), 133-143 2016

14. A. Shahzad, M. Lee, H.D. Kim, S. Woo, N. Xiong, New security development and trends to secure the SCADA sensors automated transmission during critical sessions. Symmetry 7(4), 1945-1980 (2015)

15. A. Shahzad, M. Lee, C. Lee, N. Xiong, S. Kim, Y. Lee, K. Kim, S. Woo, G. Jeong, The protocol design and new approach for SCADA security enhancement during sensors broadcasting system. Multimed. Tools Appl. 75(22), 14641 14668 (2015)

16. X. Jing, H. H. H. Yang, M.H. Au, S. Li, N. Xiong, M. Imran, A.V. Vasilakos, A quantitative risk assessment model involving frequency and threat degree under line-of-business services for infrastructure of emerging sensor networks. Sensors 17(3), 642 (2017)
17. J. Li, H. H, K. Qiao, N. Xiong, A novel topology link-controlling approach for active defense of a node in a network. Sensors 17(3), 553 (2017)

18. J. Li, N. Xiong, J.H. Park, C. Liu, M.A. Shihua, S.E. Cho, Intelligent model design of cluster supply chain with horizontal cooperation. J. Intell. Manuf. 23(4), 917-931 2009

19. N. Xiong, X. Jia, L.T. Yang, A.V. Vasilakos, Y. Li, Y. Pan, A distributed efficient flow control scheme for multirate multicast networks. IEEE Trans. Parallel Distrib. Syst. 21(9), 1254-1266 (2010)

20. Y. Yang, N. Xiong, N.Y. Chong, X. Défago, in 2008 The 3rd International Conference on Grid and Pervasive Computing - Workshops. A decentralized and adaptive flocking algorithm for autonomous mobile robots (Kunming, 2008), pp. 262-268

21. N. Xiong, A.V. Vasilakos, L.T. Yang, L. Song, Y. Pan, R. Kannan, Y. Li, Comparative analysis of quality of service and memory usage for adaptive failure detectors in healthcare systems. IEEE J. Sel. Areas Commun. 27(4) 495-509 (2009)

22. S. Rajba, P. Raif, T. Rajba, in 2013 IEEE Symposium on Computational Intelligence in Healthcare and e-Health. Wireless sensor networks in application to patients health monitoring (Singapore, 2013), pp. 94-98

23. C. Lin, N. Xiong, J.H. Park, T. Kim, Dynamic power management in new architecture of wireless sensor networks. Int. J. Commun. Syst. 22(6), 671693 (2009)

24. Y. Zeng, C.J. Sreenan, N. Xiong, L.T. Yang, J.H. Park, Connectivity and coverage maintenance in wireless sensor networks. J. Supercomput. 52(1), 23-46 (2010)

25. W. Fang, Y. Li, H. Zhang, N. Xiong, J. Lai, A.V. Vasilakos, On the throughputenergy tradeoff for data transmission between cloud and mobile devices. Inf. Sci. 283, 79-93 (2014)

26. H. Cheng, Z. Su, N. Xiong, Y. Xiao, Energy-efficient nodes scheduling algorithms for wireless sensor networks using Markov random field model. Inf. Sci. 329(C), 461-477 (2016)

27. W. M, L. Tan, N. Xiong, Data prediction, compression and recovery in clustered wireless sensor networks for environmental monitoring applications. Inf. Sci. 329(C), 800-818 (2016)

28. $\quad H$. Cheng, Y. Chen, N. Xiong, F. Li, Layer-based data aggregation and performance analysis in wireless sensor networks. J. Appl. Math. 502381, 1-12 2013

29. F. Xia, R. Hao, J. Li, N. Xiong, L.T. Yang, Y. Zhang, Adaptive GTS allocation in IEEE 802.15.4 for real-time wireless sensor networks. J. Syst. Archit. 59(10), 1231-1242 (2013)

30. A. Kurs, A. Karalis, R. Moffatt, Wireless power transfer via strongly coupled magnetic resonances. Science 317(5834), 83-86 2007

31. C. Qin, Y. Sun, Y. Zhang, in 2017 29th Chinese Control And Decision Conference. A novel path planning of mobile charger in wireless rechargeable sensor networks (Chongqing, 2017), pp. 2063-2067

32. C. Lin, R. Han, $\mathrm{P}^{2} \mathrm{~S}$ : a primary and passer-by scheduling algorithm for onremand charging architecture in wireless rechargeable sensor networks. IEEE Trans. Veh. Technol. 66(9), 8047-8058 (2017)

33. S. Zhang, W. J, L. S, Collaborative mobile charging. IEEE Trans. Comput. 64(3), 654-667 (2014)

34. M. Zhao, J. Li, Y. Yang, in Proceeding ITC'11 Proceedings of The 23rd International Teletraffic Congress. Joint Mobile Energy Replenishment and Data Gathering in Wireless Rechargeable Sensor Networks (San Francisco, 2011), pp. 238-245

35. L. Xie, Y. Shi, Y. Hou, W. Lou, H. Sherali, Multi-node wireless energy charging in sensor networks. IEEE/ACM Trans. Networking 23(2), 437-450 (2014)

36. L. Xie, Y. Shi, Y.T. Hou, H.D. Sherali, Making sensor networks immortal: an energy-renewal approach with wireless power transfer. IEEE/ACM Trans. Networking 20(6), 1748-1761 (2012)

37. S. He, J. Chen, F. Jiang, D. Yau, G. Xing, Y. Sun, Energy provisioning in wireless rechargeable sensor networks. IEEE Trans. Mob. Comput. 12(10), 1931-1942 (2013)

38. P. Wu, F. Xiao, C. Sha, H. Huang, R. Wang, N. Xiong, Node scheduling strategies for achieving full-view area coverage in camera sensor networks. Sensors 17(6), 1303 (2017)

39. W. Chien, H. Cho, H. Chao, in 2016 International Wireless Communications and Mobile Computing Conference. Enhanced SA-based charging algorithm for WRSN (Paphos, 2016), pp. 1012-1017

40. G. Sun, Y. Liu, M. Yang, A. Wang, Y. Zhang, in IEEE Global Communications Conference. Charging nodes deployment optimization in wireless rechargeable sensor network (Singapore, 2017), pp. 1-6

41. J. Liao, J. Jiang, in 2014 7th International Conference on Ubi-Media Computing and Workshops. Wireless charger deployment optimization for wireless rechargeable sensor networks (Ulaanbaatar, 2014), pp. 160-164 
42. J.H. Liao, C.M. Hong, J.R. Jiang, An adaptive algorithm for charger deployment optimization in wireless rechargeable sensor networks. Front. Artif. Intell. Appl. 274, 2080-2089 (2015)

43. W. Cheng, M. Zhao, N. Xiong, K.T. Chui, Non-convex sparse and low-rank based robust subspace segmentation for data mining. Sensor 17(7), 1633 (2017)

44. L. RW Liu, S.C.H.Y. Shi, N. Xiong, D. Wang, Reconstruction of undersampled big dynamic MRI data using non-convex low-rank and sparsity constraints. Sensors 17(3), 509 (2017)

45. S. Wang, H. Yi, W. L, F. Zhou, N. Xiong, Mining probabilistic representative gathering patterns for mobile sensor data. J. Int. Technol. 18(2), 321-332 (2017)

46. Q. Fan, N. Xiong, K. Zeitouni, Game balanced multi-factor multicast routing in sensor grid networks. Inf. Sci. 367, 550-572 (2016)

47. Q. Fan, K. Zeitouni, N. Xiong, W. Q, A General Nash Equilibrium Semantic Cache Algorithm in a Sensor Grid Database System. International Conference on Data Management in Cloud, Grid and P2P Systems, vol 8648 (2014), pp. 13-24

48. T.L. Lin, S.L. Li, H.Y. Chang, A power balance aware wireless charger deployment method for complete coverage in wireless rechargeable sensor networks. Energies 9(9), 695 (2016)

49. R.G. Michael, S.J. David, Computers and Intractability: A Guide to the Theory of NP-Completeness (W. H. Freeman and Company, New York, 1979), pp. 187-288

50. N. Nguyen, B. Liu, The mobile sensor deployment problem and the target coverage problem in mobile wireless sensor networks are NP-hard. IEEE Syst. J. 9(99), 1-4 (2018)

51. M. Cardei, J. Wu, Energy-efficient coverage problems in wireless ad-hoc sensor networks. Comput. Commun. 29(4), 413-420 (2006)

52. L. Li, D. Wang, X. Shen, M. Yang, in 2009 International Conference on Computational Intelligence and Software Engineering. A method for combinatorial explosion avoidance of Al planner and the application on test case generation (Wuhan, 2009), pp. 1-4

53. C. Chung, A. Matsuoka, Y. Yang, in IEEE/ACM 5th International Workshop on Games and Software Engineering. Serious games for NP-hard problems: challenges and insights (Austin, 2016), pp. 29-32

54. L.V. Ahn, Games with a purpose. Computer 39(6), 92-94 (2006)

55. K.S. Hung, K.S. Lui, On perimeter coverage in wireless sensor networks. IEEE Trans. Wirel. Commun. 9(7), 2156-2164 (2010)

\section{Submit your manuscript to a SpringerOpen ${ }^{\circ}$ journal and benefit from:}

- Convenient online submission

- Rigorous peer review

- Open access: articles freely available online

- High visibility within the field

- Retaining the copyright to your article

Submit your next manuscript at $\boldsymbol{\nabla}$ springeropen.com 